\title{
Stakeholder views of breastfeeding education in schools: a systematic mixed studies review of the literature
}

\author{
Nicola Singletary ${ }^{*}$ (D) Ellen Chetwynd, L. Suzanne Goodell and April Fogleman
}

\begin{abstract}
Background: Breastfeeding provides numerous health benefits for mothers and infants, but worldwide breastfeeding rates fall below recommendations. As part of efforts to increase breastfeeding initiation and duration, the World Health Organization and UNICEF UK recommend educational interventions to increase awareness and positive attitudes towards breastfeeding beginning during the school years. Breastfeeding education in the school setting offers the opportunity to improve the knowledge base, address misconceptions, and positively influence beliefs and attitudes for students from a wide range of socioeconomic and cultural backgrounds. The purpose of this paper is to present a comprehensive narrative review of the literature regarding student and teacher (stakeholder) views of breastfeeding and breastfeeding education programs in schools to inform future research in the area.

Methods: Articles were located through a systematic search of online databases and journals using the following keywords in various combinations: (1) breastfeeding, lactation, breast-feeding, "bottle feeding", "infant feeding" (2) student, educator, teacher, "school administrator" and (3) schools, "secondary education", "primary education", "K-12", "high school", "middle school", "elementary school", education, adolescents, curriculum, and a manual search of article references. Studies were screened for inclusion against specific criteria and included papers were assessed using the Mixed Methods Appraisal Tool (MMAT).

Results: This review suggests that adolescents have a deficit in breastfeeding knowledge and express negative conceptions about breastfeeding. Breastfeeding is being discussed in some school environments, but the extent of lessons and the specific messages that teachers communicate have not been explored. Students appear to be interested in receiving more information about breastfeeding, especially if delivered by health professionals or breastfeeding mothers. The majority of teachers are supportive of incorporating breastfeeding education in family and consumer sciences, sexual education, and health classes; however, time constraints and limited knowledge of infant feeding recommendations may be barriers to implementation of appropriate lesson plans.
\end{abstract}

Conclusions: Students generally support and are receptive to breastfeeding education; however, research on educator attitudes, knowledge, and experiences are necessary for appropriate implementation of breastfeeding education in varying school settings around the world.

Keywords: Breastfeeding, Infant feeding, Education, Schools, Stakeholders, Students, Teachers

\footnotetext{
* Correspondence: nsingle@ncsu.edu

Department of Food, Bioprocessing, and Nutrition Sciences, North Carolina

State University, Box 7624, Raleigh, NC 27695-7624, USA
}

\section{Biomed Central}

(c) The Author(s). 2017 Open Access This article is distributed under the terms of the Creative Commons Attribution 4.0 International License (http://creativecommons.org/licenses/by/4.0/), which permits unrestricted use, distribution, and reproduction in any medium, provided you give appropriate credit to the original author(s) and the source, provide a link to the Creative Commons license, and indicate if changes were made. The Creative Commons Public Domain Dedication waiver (http://creativecommons.org/publicdomain/zero/1.0/) applies to the data made available in this article, unless otherwise stated. 


\section{Background}

The health benefits of breastfeeding for both mother and baby are well established [1-3]. The World Health Organization (WHO) and the American Academy of Pediatrics (AAP) recommend exclusive breastfeeding for the first 6 months of life, with continued breastfeeding into and beyond the second year [1, 4]. However, global rates for initiation and duration of breastfeeding fall below these recommendations [3].

In an attempt to increase breastfeeding initiation and duration, global and national policy documents advocate for increased breastfeeding support in our society [4-6]. In the United States, The Surgeon General's Call to Action to Support Breastfeeding recommends education to help increase knowledge, skills, and positive attitudes regarding breastfeeding, emphasizing appropriate support of the mother from her family, health care providers, and community [6]. Likewise, the World Health Organization endorses strategies that allow parents to make informed decisions about infant feeding through the use of evidence-based educational materials that are designed without commercial influence [4]. In particular, this document calls for schools and programs that work with children and adolescents to provide education that promotes awareness and positive attitudes towards breastfeeding as part of the general curriculum [4]. The UNICEF UK Baby Friendly Initiative also recommends breastfeeding education in schools for both male and female students [5]. According to this initiative, providing schoolaged children with information about breastfeeding would enable them to make informed choices about infant feeding when they become parents [5].

Women make the decision to breastfeed or formula feed either before or during the early weeks of pregnancy and maintain those decisions throughout their pregnancy [7]. Furthermore, many children and adolescents have already considered infant feeding choices for when they become parents [8-11]. Based on these early decisions regarding infant feeding, efforts to promote breastfeeding during the prenatal or immediate postpartum period may not be effective. Introducing breastfeeding education in the school setting presents a unique opportunity to engage both male and female students from a variety of socioeconomic and cultural backgrounds early in their decision-making process.

In light of the global focus on infant feeding, educational initiatives for school-aged children have the potential to normalize breastfeeding for all segments of the population; however, successful interventions require enabling conditions. Understanding the views and knowledge of stakeholders (educators and students) directly involved with targeted school systems is a critical first step before designing and implementing educational interventions and policies that support breastfeeding. The purpose of this article is a systematic review of the quantitative and qualitative research in order to understand stakeholder knowledge regarding breastfeeding and attitudes towards breastfeeding education in schools. The results of the review are organized as a narrative synthesis discussing the research for each stakeholder group: teachers and students.

\section{Methods}

\section{Search strategy}

A librarian-assisted literature search was conducted by the first author using three online databases: Web of Science, EBSCOhost Research Databases, and PubMed. The last search was conducted on October 7, 2016. Relevant search terms included the following: (1) breastfeeding, lactation, breast-feeding, "bottle feeding", "infant feeding" (2) student, educator, teacher, "school administrator" and (3) schools, "secondary education", "primary education", "K-12", "high school", "middle school", "elementary school", education, adolescents, curriculum. Additional articles were located using a manual search of the references of studies from the database search, articles that were suggested electronically during the search online, and online archives of journals related to human lactation and health education.

\section{Screening strategy}

During the first phase of screening, the titles and abstracts of the studies identified through the search were screened for inclusion criteria. To be included, articles had to (1) address stakeholder views of breastfeeding or breastfeeding education in schools, (2) be published between 1990 and October 2016, 3) be published in peer-reviewed journals, (4) present original research, and (5) have full text in English. There were no restrictions on the country of study. During the second phase of screening, full text studies were retrieved and assessed according to the same inclusion criteria by two independent reviewers (NS and EC). Disagreements were discussed until consensus was reached.

\section{Search outcome}

The database search identified 2583 hits (1993 EBSCO, $210 \mathrm{Web}$ of Science, 380 PubMed) and hand searching identified an additional 24 records. After screening the titles and abstracts for inclusion criteria and removing duplicates, 71 articles remained. Full-text examination of the articles left 48 articles for inclusion in this review Fig. 1). Of the 48 articles included, 39 were quantitative studies, one was qualitative and eight were mixed methods studies. Due to the mixed nature of the studies, a mixed studies systematic review process was chosen for this review. This type of review is ideal for synthesizing research of varying methodologies while remaining 


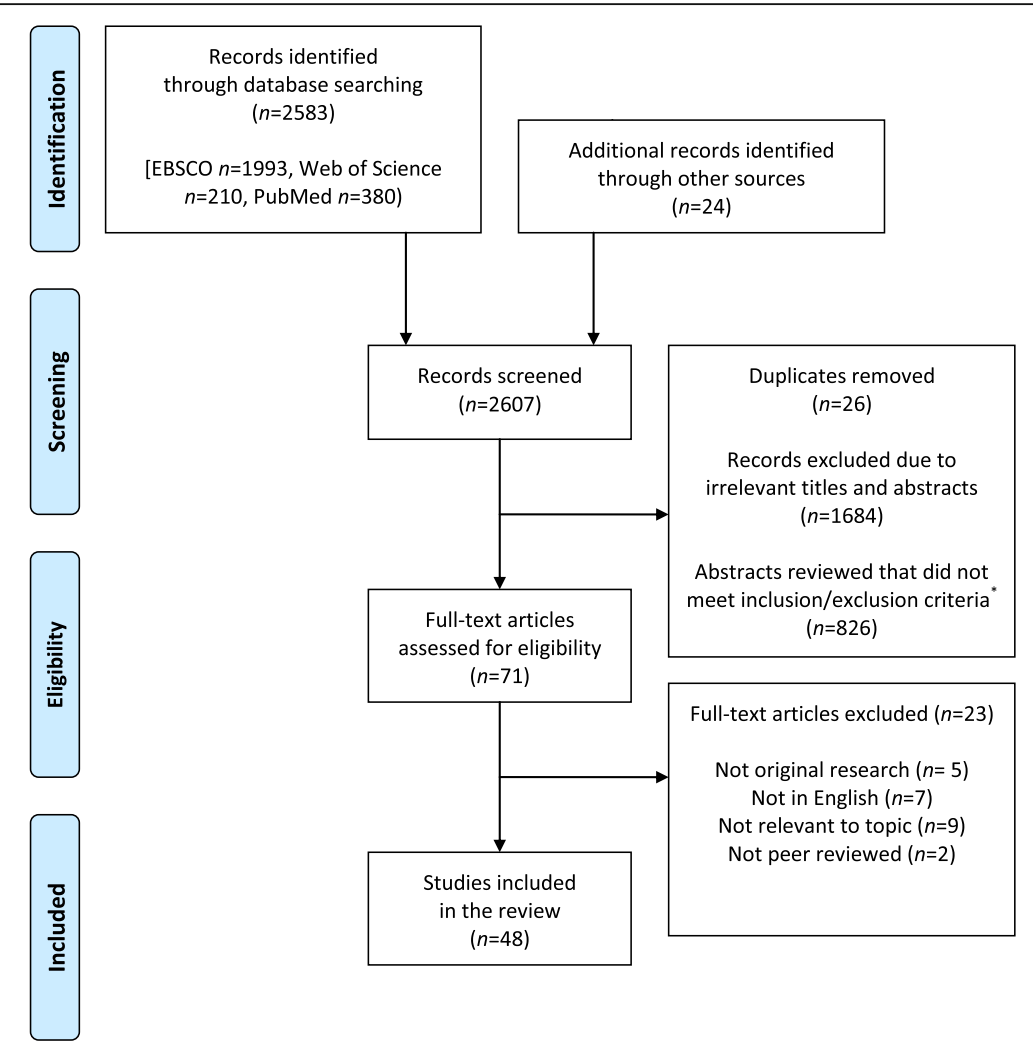

Fig. 1 PRISMA (Preferred Reporting Items for Systematic Reviews and Meta-Analyses) flow diagram of literature search

sensitive to the context of the research across complex fields [12].

\section{Quality appraisal of studies}

The Mixed Methods Appraisal Tool (MMAT) was used for quality assessment of the articles [13]. This quality appraisal tool has been tested for validity, efficiency, and reliability for use with qualitative, quantitative, and mixed methods studies [12]. Two independent reviewers (NS and EC) appraised each study and disagreements were discussed until consensus was reached (Tables 1, 2 and 3).th=tlb=

\section{Results and Discussion}

Teacher knowledge of breastfeeding and views regarding breastfeeding education

The willingness and ability of teachers to incorporate infant feeding education in their classrooms is a critical component of research on school breastfeeding education program development and implementation. Seven studies examined teachers' knowledge of breastfeeding [14-20] and two investigated their views on incorporating breastfeeding education into schools $[14,21]$.

Teachers' knowledge of and attitudes toward infant feeding recommendations and practices have implications on their willingness and ability to present breastfeeding education to their students. The research that has been done indicates that teachers have a basic knowledge of the benefits of breastfeeding but are not aware of or have misconceptions about specific infant feeding recommendations. In 2004, a study was conducted in Nigeria of 84 home economics teachers. The study was designed to assess teacher awareness of breastfeeding and the Baby Friendly Initiative (BFI). Researchers found that $70.2 \%$ of teachers surveyed knew that human milk can prevent malnutrition and 53.6\% agreed that breastfeeding should continue for at least 2 years. Beyond this knowledge, the teachers' understanding was low in regards to the concepts of the BFI, the benefits of colostrum, feeding on demand, and protection of breastfeeding by the law [14]. Similarly, in a 1990 study of infant feeding knowledge among 100 newly graduated Indian teachers, researchers found that the majority identified human milk as the best way to feed infants and that babies should be breastfed for over 1 year. Of those surveyed, 77\% knew that breastfeeding is beneficial for the mother, and $90 \%$ recognized that breastfeeding is important in mother-child bonding. However, many had misconceptions about infant feeding best practices, including $61 \%$ agreeing that first feeds other than colostrum are appropriate and $100 \%$ believing that milk needs to be diluted with water so it is not too heavy for the infant's stomach [16]. In Pakistan, the breastfeeding 


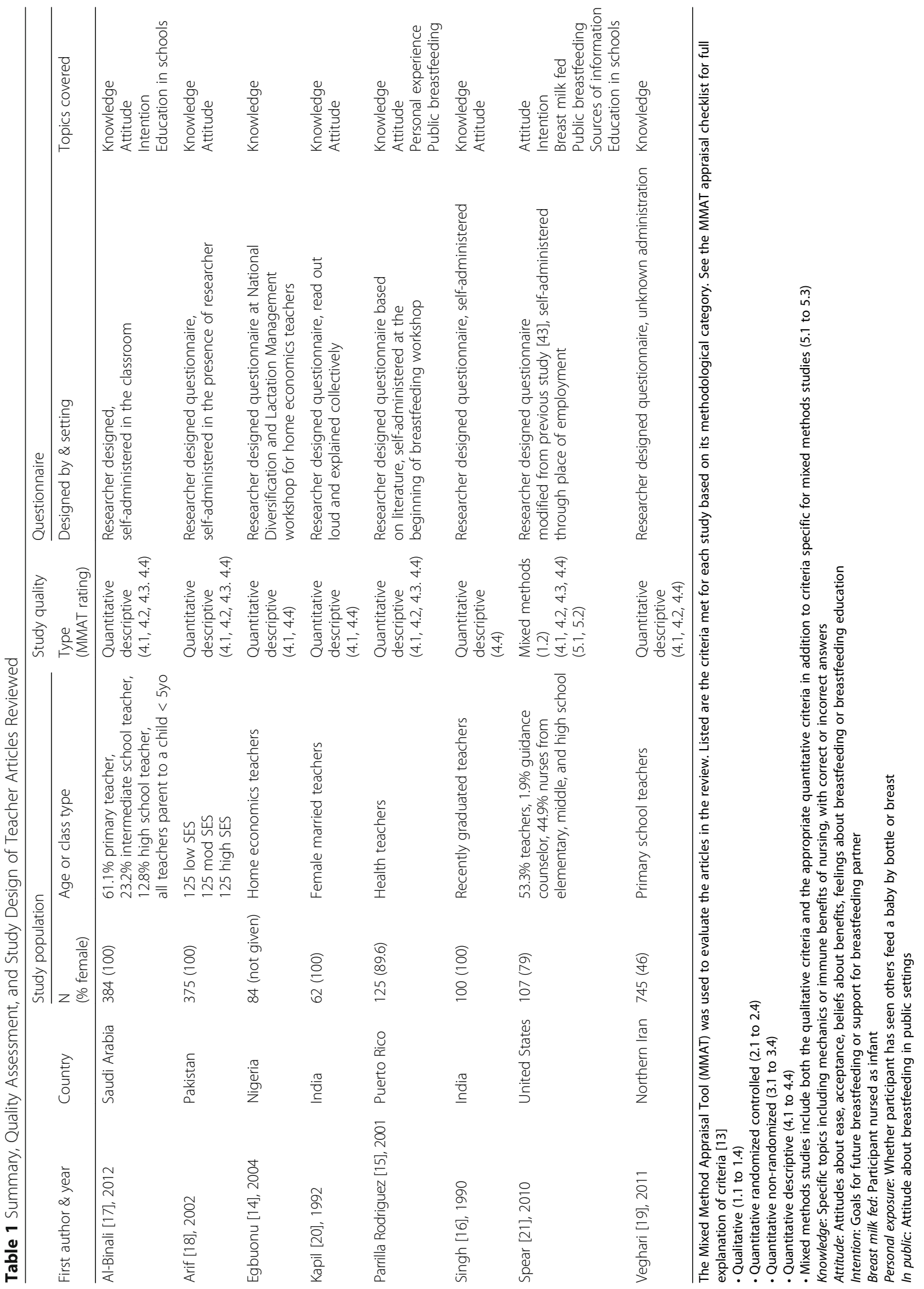




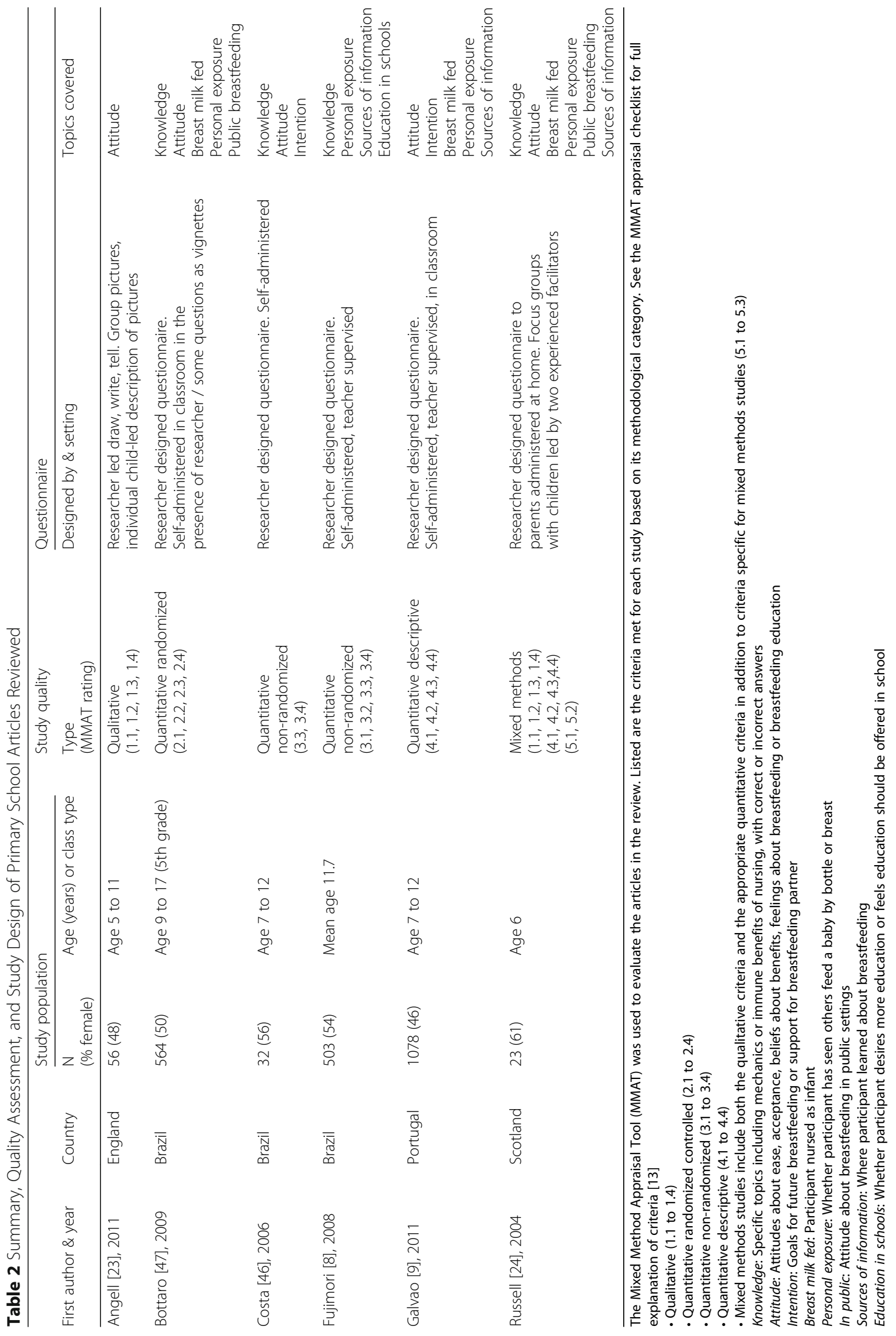




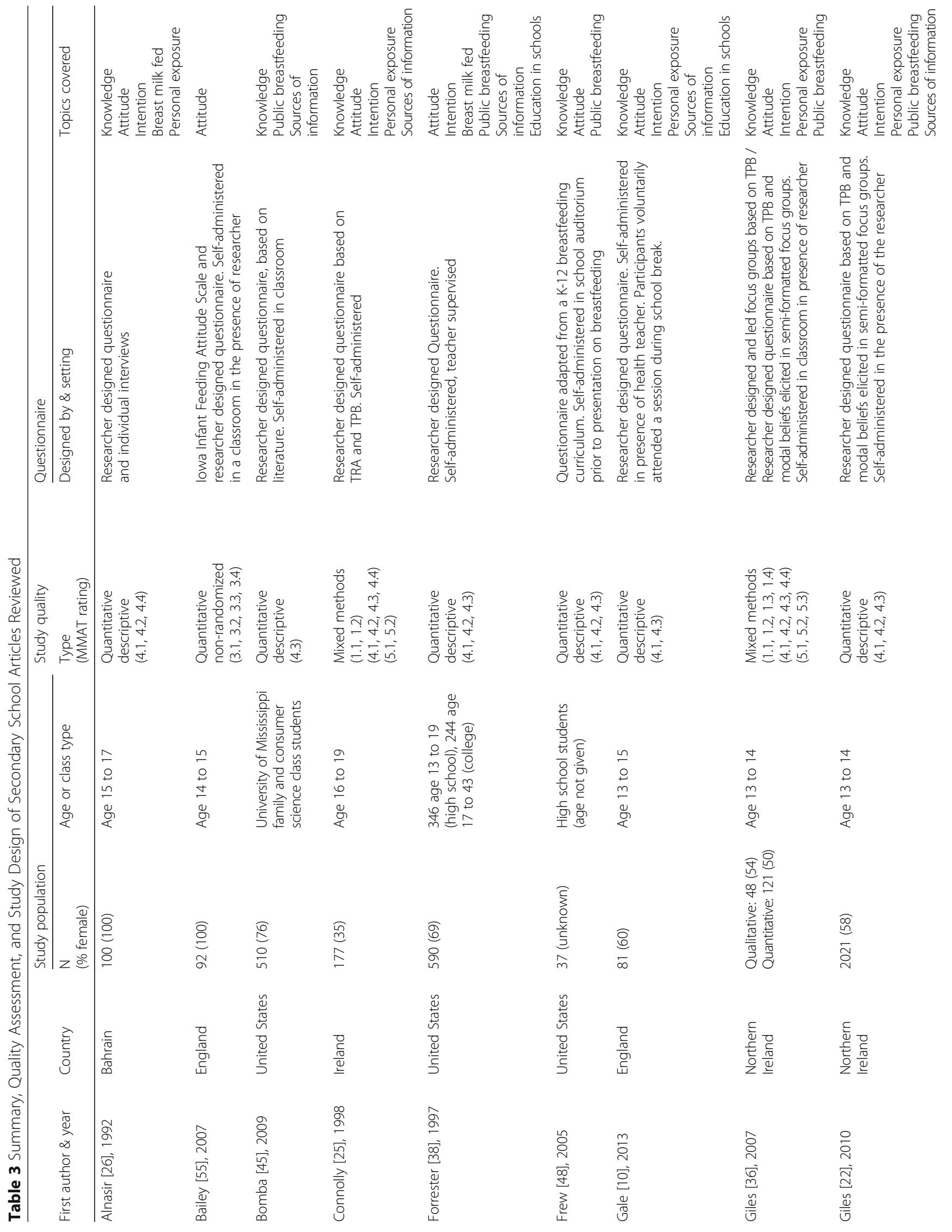




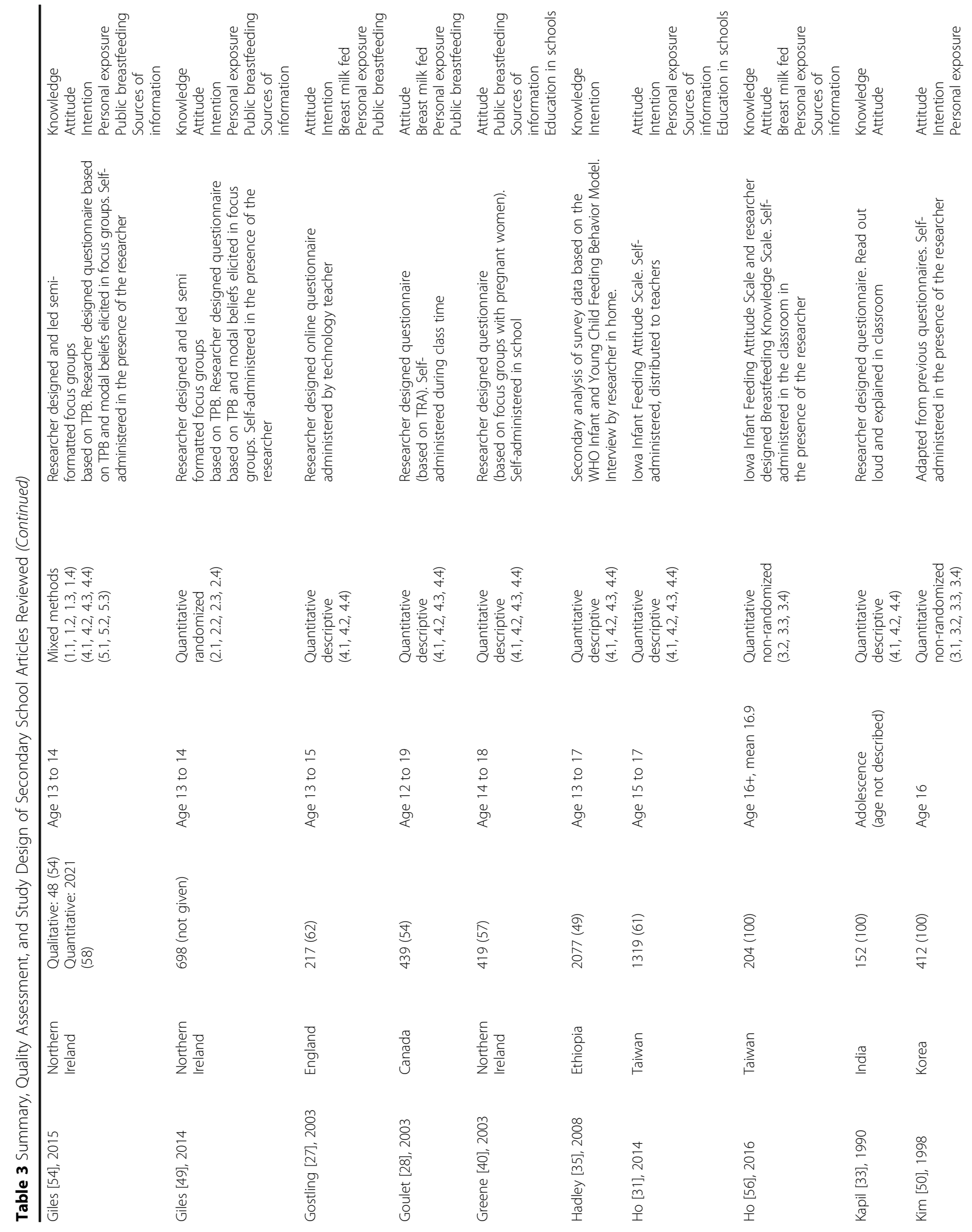




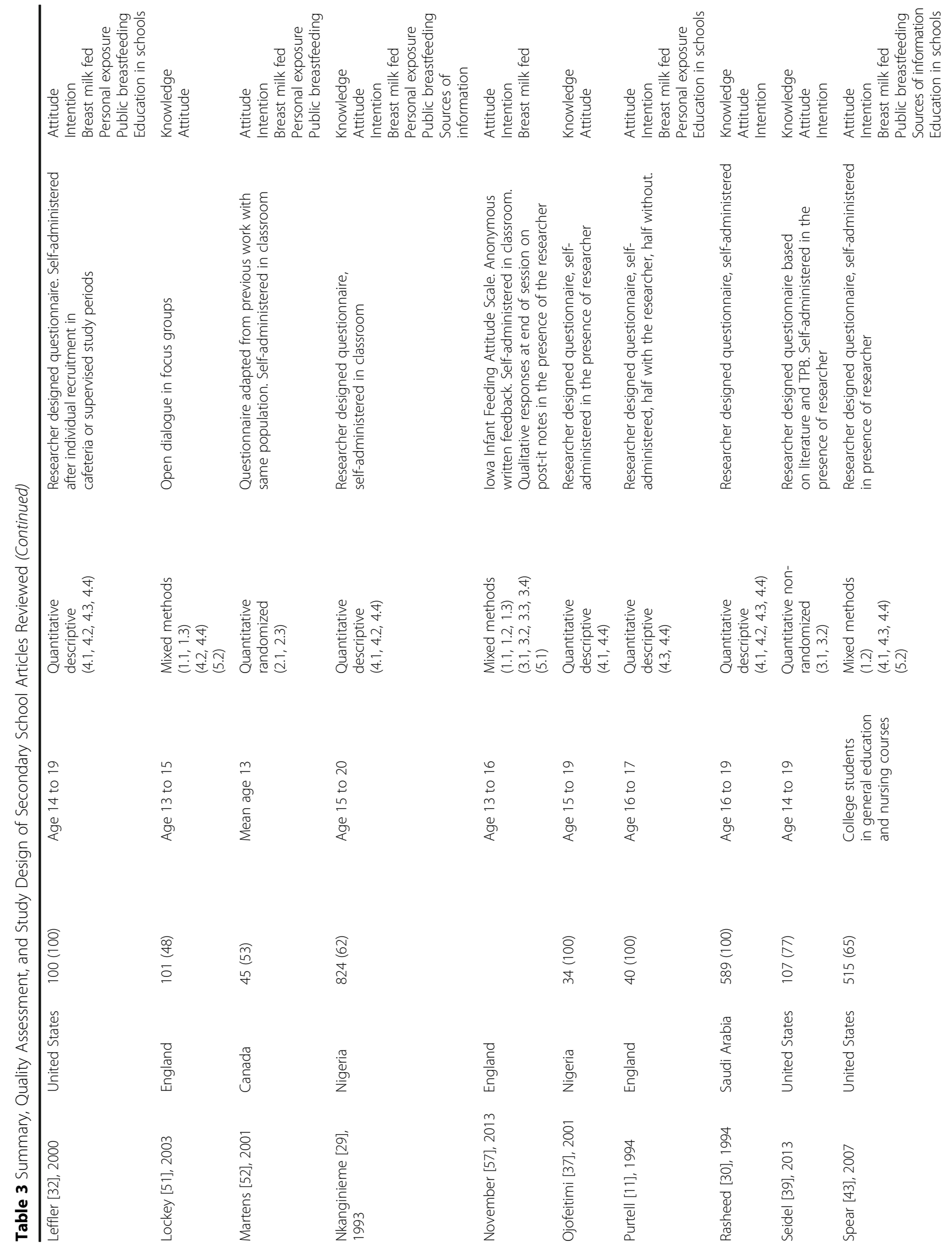




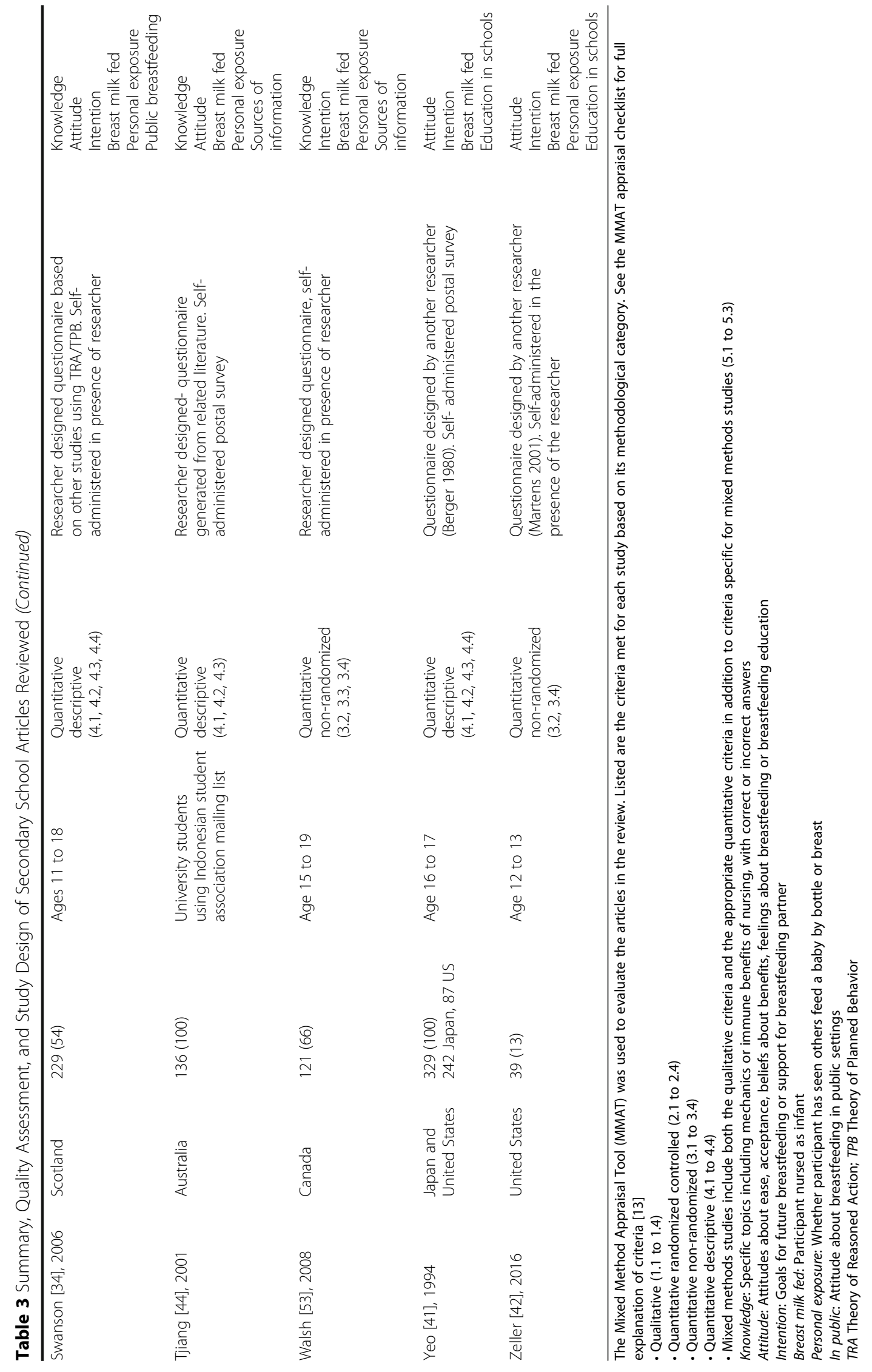


knowledge of 375 female school teachers from different socioeconomic areas was assessed. The majority of teachers in each socioeconomic group knew that breastfeeding on demand is preferred and that mothers need additional food when breastfeeding, but less than one fourth knew that babies should only be given breast milk for the first 6 months of life and that breastfeeding should be continued to 18-24 months of life [18]. Research with 384 female teachers who had a child 5 years of age or less in Saudi Arabia indicated that $89.3 \%$ knew the benefits of colostrum, but only $28 \%$ identified that a child should receive only breast milk for the first 6 months of life [17]. In northern Iran, 745 male and female primary school teachers completed a questionnaire about infant feeding. Overall, $81.6 \%$ agreed that breastfeeding is beneficial during the first 6 months of life, but $17.6 \%$ also considered other milk or milk substitutes appropriate [19]. Furthermore, a study in 2001 of 125 Puerto Rican health teachers showed that 96.7\% agreed that breastfeeding helps establish a bond between mother and child, $98.4 \%$ agreed it protects the baby against infections, and $98.4 \%$ agreed it provides the best nutrition for the baby. However, $60.3 \%$ agreed that mothers must follow a specific diet when breastfeeding and 36\% thought that breastfeeding should be alternated with formula [15]. These studies indicate that teachers may have generally positive attitudes towards breastfeeding and knowledge of the basic benefits of breast milk feeding, but they have limited knowledge of and incorrect beliefs about specific infant feeding recommendations.

Previous research on teachers' attitudes toward including breastfeeding education in schools indicates that the majority of teachers are supportive of including breastfeeding education in the classroom [14, 21], but they have limited time to include this material in the curriculum [21]. The Nigerian study by Egbuonu et al. concluded that the majority of the 84 home economics teachers surveyed were supportive of teaching about the BFI in primary, secondary, and tertiary education [14]. Similar research in the United States conducted in 2010 showed that $86.9 \%$ of the 107 teachers and school nurses surveyed thought that the benefits of breastfeeding should be incorporated into high school curricula, and $57.9 \%$ believed the topic should be taught at the middle school level. The teachers and school nurses identified that the following classes would be the most appropriate subjects in which to incorporate breastfeeding content: $34.6 \%$ family life, $11.2 \%$ health, $11.2 \%$ sex education, and $8.4 \%$ science. In addition, the majority of those surveyed agreed that it is important to promote a breastfeeding culture in the United States and that breastfeeding in public is acceptable. While the majority agreed that breastfeeding education should be included in the curriculum, only $15.9 \%$ were teaching about the benefits of breastfeeding, $5.6 \%$ at the middle school level and $10.3 \%$ at the high school level. Reasons cited for not teaching about breastfeeding include limited time and lack of breastfeeding education in the required curriculum. A minority of teachers and nurses believed that breastfeeding should not be taught in the classroom (13\% at the high school level and $42 \%$ at middle school level) despite agreeing that all mothers should hear about the distinctive benefits of breastfeeding from their health care providers. Open response comments ranged from "Breastfeeding should be strongly encouraged" to "Students should be educated about breastfeeding, but breastfeeding should not be endorsed" (pg. 142) [21]. Although these two studies covered a limited geographical area, they suggest that teachers may be willing to incorporate the benefits of breastfeeding into family and consumer science, health, or science classes if it is part of the required curriculum or could easily be integrated with existing curriculum goals and lessons. Despite research that many teachers support including breastfeeding education in the general curriculum, some teachers and administrators feel that breastfeeding content is only suitable for female students [22] and are concerned that breastfeeding education is a sensitive subject closely linked to teen pregnancy [21, 22]. In addition, researchers have met reluctance from school administrators when attempting to conduct research on breastfeeding education within schools. Administrators cited concerns regarding the appropriateness of the subject matter for the age or gender of the students [21].

\section{Student knowledge of breastfeeding and views regarding breastfeeding education}

Understanding the views of students regarding breastfeeding and breastfeeding education is an important part of designing appropriately targeted educational interventions. With this knowledge, educators can develop and implement lessons and curricula that are studentcentered and focus on the areas of greatest need and potential impact. Due to the differences in school age classification internationally, for this review we define primary as age 11 or younger and secondary as ages 12-19 unless otherwise indicated in Tables 2 and 3.

In the primary grades, some girls and boys are aware of breastfeeding through exposure to infant feeding in their family or community, but many have already internalized bottle feeding and formula as the norm for infant feeding $[8,9,23,24]$. The results of research on the attitudes toward breastfeeding for secondary male and female students are mixed. Some studies demonstrate positive attitudes towards breastfeeding [25-30], while other results are neutral or negative [25, 31]. Even students with positive attitudes towards breastfeeding can lack specific knowledge of breastfeeding and infant 
feeding recommendations $[8,9,22,25,29,30,32-37]$. Many students know that breastfeeding has health benefits for babies [9-11, 25, 26, 28, 33, 36, 38]; however, most children are not aware of the specific benefits of breastfeeding for the infant or for the mother [8-11, 22, 33, 34, 36, 39]. Furthermore, students have misconceptions that breastfeeding can have a negative impact on the mother's health and lifestyle $[9,10,33]$.

Primary [23, 24] and secondary [10, 11, 27, 31, 38, 40,41] school children are receptive to breastfeeding education in schools. Secondary school students also recognize that education has the potential to increase awareness and knowledge of breastfeeding and help normalize breastfeeding $[10,27,38,42]$. Inclusion of breastfeeding education in the school setting is limited. In a survey of 515 United States college students, 36.7\% remembered being taught about breastfeeding during high school and only $11 \%$ recalled lessons on the topic during middle school [43]. Comparatively, only $6.6 \%$ of 136 female students in Indonesia recalled learning about breastfeeding in school [44]. In similar research in the United States, England, Northern Ireland, and Taiwan, approximately one fourth of secondary students reported receiving information about breastfeeding in high school [10, 31, 38, 40, 45]. In contrast, $52 \%$ of girls in Bahrain and $48.6 \%$ of students in Nigeria recalled being taught about infant feeding in school $[26,29]$.

There is an emerging body of work that shows that interventions with children $[8,46,47]$ and adolescents $[39,42,48-57]$ in the school environment have the potential to positively affect their breastfeeding attitudes and knowledge. In research by Greene et al. in Northern Ireland, 76\% of secondary students surveyed agreed that information about breastfeeding should be part of the main curriculum, with $88 \%$ agreeing it should be part of the child development module, $78 \%$ part of sex education, and $62 \%$ part of home economics [40]. However, some adolescents are unsure whether teachers have adequate knowledge to teach this information and may prefer that lessons be taught by a health care provider or a breastfeeding mother $[11,40]$.

Surveys of college students in the United States also show support for breastfeeding education at the secondary school level $[43,45]$. In a 2007 study by Spear, the majority of the college students surveyed believed the benefits of breastfeeding should be included in the high school curriculum $(87.2 \%$ agreed or strongly agreed and $12.8 \%$ disagreed or strongly disagreed) but only one third thought it should be included in the middle school curriculum (34.9\% agreed or strongly agreed and $65.1 \%$ disagreed or strongly disagreed) [43]. Another survey of college students showed that they felt breastfeeding education was more acceptable for students at the high school level compared to the middle school level and more so for girls than boys (78\% high school girls, $42 \%$ high school boys, 32\% middle school girls and 15\% middle school boys). In addition, $45 \%$ of respondents identified that high school teachers are an important source of infant feeding practices education [45].

\section{Conclusions}

This review provides evidence that primary and secondary school teachers are willing to incorporate infant feeding education into the classroom and many understand the basic benefits of breastfeeding. Teacher support enables the development and implementation of breastfeeding education programs as a vital component of breastfeeding promotion initiatives. Additional research into the attitudes, knowledge, and experiences of educators regarding teaching breastfeeding in schools would add to our understanding of how best to implement lessons and curricula in the future. Areas of study should include teachers' views on barriers to implementing breastfeeding education in schools, teachers' comfort with the content and knowledge of breastfeeding, the best way to address gaps in teacher knowledge, and the replication of teacher acceptability of breastfeeding education in a variety of geographic areas.

This review indicates that breastfeeding is being discussed in some school environments, but the research is limited geographically. Furthermore, the extent and specific messages children receive have not been explored. In many cases, students are interested in receiving more information about breastfeeding, especially if it comes from health professionals or breastfeeding mothers. It is imperative that the education children get from school provides unbiased information about current feeding recommendations, enabling them to make informed decisions when they become parents. It is also critical to consider the views of other major stakeholders when designing and implementing educational programs that address breastfeeding. Researchers indicated that they encountered resistance from school administrators, but there is very little research on the views of administrators regarding breastfeeding education in schools.

A limitation of this review is that it does not include research from unpublished dissertations and theses or studies published in languages other than English. Despite this limitation, this review suggests that breastfeeding education in the school setting offers the opportunity to introduce the topic to a wide range of students from a variety of socioeconomic and cultural backgrounds around the world. By introducing the topic in schools we can build knowledge and positive ideas 
throughout childhood and adolescence that can be carried into adulthood regardless of a persons' ethnicity, culture, education, or income. Incorporating positive breastfeeding messages as part of the health, science, and family and consumer science curricula in schools will promote a society that is supportive of breastfeeding.

Existing research offers us information that can be used to develop targeted educational programs for primary and secondary schools. These programs should work towards improving awareness of breastfeeding in general and address areas shown as lacking in student knowledge such as the specific benefits of breastfeeding for the mother, the infant, and their relationship. Well-crafted lessons have the potential to increase understanding of the importance of breastfeeding and dispel myths that breastfeeding has a negative impact on the health and well-being of the mother. These programs are needed to address negative attitudes toward breastfeeding and to respond to students' receptiveness to more information on the subject, enabling them to make informed decisions as they enter adulthood and parenthood. Correcting misconceptions about breastfeeding is beneficial for future parents and public health in general, creating a more accepting and supportive culture for breastfeeding.

\section{Acknowledgements}

We would like to thank Mohan Ramaswamy, MSc(Ag), MLIS, PhD for his assistance with the literature search

\section{Funding}

Not applicable.

\section{Availability of data and material}

Not applicable.

\section{Authors' contributions}

NS conducted the literature search, reviewed the research articles for inclusion/exclusion and quality appraisal and drafted the manuscript. EC reviewed the research articles for inclusion/exclusion and quality appraisal, drafted Tables 1, 2 and 3, and reviewed the manuscript. SG provided manuscript guidance and feedback. AF provided manuscript editing, guidance, and feedback. All authors read and approved the final manuscript.

\section{Competing interests}

The authors declare that they have no competing interests.

\section{Consent for publication}

Not applicable.

Ethics approval and consent to participate Not applicable.

\section{Publisher's Note}

Springer Nature remains neutral with regard to jurisdictional claims in published maps and institutional affiliations.
Received: 15 July 2016 Accepted: 15 March 2017

Published online: 27 March 2017

\section{References}

1. Gartner LM, Morton J, Lawrence RA, Naylor AJ, O'Hare D, Schanler RJ, Eidelman Al. Breastfeeding and the use of human milk. Pediatrics. 2005;115(2):496-506.

2. Horta BL, Bahl R, Martines JC, Victora CG. Evidence on the long-term effects of breastfeeding: systematic reviews and meta-analyses. Geneva: World Health Organization; 2007.

3. Victora CG, Bahl R, Barros AJ, França GV, Horton S, Krasevec J, Murch S, Sankar MJ, Walker N, Rollins NC. Breastfeeding in the 21st century: epidemiology, mechanisms, and lifelong effect. Lancet. 2016;387(10017):475-90.

4. WHO/UNICEF. Global strategy for infant and young child feeding. Geneva: World Health Organization; 2003.

5. UNICEF UK Baby Friendly Initiative. Towards national, regional and local strategies for breastfeeding. UK: UNICEF; 1999.

6. US Department of Health and Human Services. The Surgeon General's call to action to support breastfeeding. Washington, DC: US Dept. of Health and Human Services, Office of the Surgeon General; 2011.

7. Earle S. Why some women do not breast feed: bottle feeding and fathers' role. Midwifery. 2000;16(4):323-30.

8. Fujimori M, Morais TC, França EL, de Toledo OR, Honório-França AC. The attitudes of primary school children to breastfeeding and the effect of health education lectures. J Pediatr (Rio J). 2008;84(3):224-31.

9. Galvao DM, da Silva IA. Portuguese school children breastfeeding experiences. Rev Esc Enferm USP. 2011:45(5):1055-62. Portuguese.

10. Gale L, Davies N. Young people's attitudes towards breastfeeding: a survey of 13-15-year-old pupils in a south London school. Br J Midwifery. 2013;21(3):195-201.

11. Purtell M. Teenage girls' attitudes to breastfeeding. Health Visit. 1994;67(5):156-7.

12. Pluye $\mathrm{P}$, Hong QN. Combining the power of stories and the power of numbers: mixed methods research and mixed studies reviews. Annu Rev Public Health. 2014;35(1):29-45

13. Pluye P, Robert E, Cargo M, Bartlett G, O'Caithain A, Griffiths F, Boardman F, Gagnon MP, Rousseau MC. Proposal: a mixed-methods appraisal tool for systematic mixed study reviews. 2014. Available at: http:// mixedmethodsappraisaltoolpublic.pbworks.com. Accessed 4 Nov 2016.

14. Egbuonu I, Ezechukwu CC, Chukwuka JO. Level of awareness of the baby friendly initiative among home economics teachers in Nigeria. J Trop Pediatr. 2004;50(6):331-3.

15. Parrilla Rodriguez AM, Davila Torres R, Gorrin Peralta JJ, Alonso AA. Puerto Rican health teachers: attitudes towards breastfeeding. P R Health Sci J. 2001;20(1):57-61.

16. Singh $H$, Kaur $L$. Awareness about infant feeding among young lady teachers. Indian Pediatr. 1990;27(8):861-3.

17. Al-Binali AM. Breastfeeding knowledge, attitude and practice among school teachers in Abha female educational district, southwestern Saudi Arabia. Int Breastfeed J. 2012;7(1):10.

18. Arif MA, Mehdi Z. Knowledge and attitude of school teachers regarding breast-feeding. Pakistan Journal of Medical Sciences. 2002;18(2):99-107.

19. Veghari G, Mansourian A. The study of breastfeeding knowledge among northern Iranian teachers. Journal of Nepal Paediatric Society. 2011;31(1):39-43.

20. Kapil U, Bhasin S. Perception towards breast feeding amongst working women teachers of a public school in Delhi. Indian Pediatr. 1992;29(6):753-6.

21. Spear HJ. School nurses and teachers: attitudes regarding inclusion of breastfeeding education in school curricula. J Sch Nurs. 2010;26(2):137-46.

22. Giles M, Connor S, McClenahan C, Mallet J. Attitudes to breastfeeding among adolescents. J Hum Nutr Diet. 2010;23(3):285-93.

23. Angell C, Alexander J, Hunt JA. How Are babies Fed? a pilot study exploring primary school children's perceptions of infant feeding. Birth. 2011;38(4):346-53.

24. Russell B, Richards $H$, Jones A, Hoddinott P. 'Breakfast, lunch and dinner': attitudes to infant feeding amongst children in a Scottish primary school. A qualitative focus group study. Health Educ J. 2004;63(1):70-80.

25. Connolly C, Kelleher CC, Becker G, Friel S, Gabhainn SN. Attitudes of young men and women to breastfeeding. Ir Med J. 1998;91(3):88-9. 
26. Alnasir FA. Knowledge and attitude of secondary school-girls towards breast-feeding in Bahrain. J Bahrain Med Soc. 1992:4(1):6-10.

27. Gostling L. Breastfeeding through the eyes of the teenager. MIDIRS Midwifery Digest. 2003;13(4):549-56.

28. Goulet C, Lampron A, Marcil I, Ross L. Attitudes and subjective norms of male and female adolescents toward breastfeeding. J Hum Lact. 2003;19(4):402-10.

29. Nkanginieme KEO, Ibe BC. Experiences, attitudes and beliefs of secondary school students about breastfeeding. Niger J Paediatr. 1993;20(2):35-40.

30. Rasheed P. Perception of infant feeding practices among mothers-to-be: an urban-based school study. J Family Community Med. 1994;1(1):72-8.

31. Ho YJ, Yu CC. Attitudes of high school and vocational school students toward breastfeeding in Taiwan. J Perinat Educ. 2014;23(2):89-95.

32. Leffler D. U.S. high school age girls may be receptive to breastfeeding promotion. J Hum Lact. 2000;16(1):36-40.

33. Kapil U, Bhasin S, Manocha S. Knowledge and attitude amongst well-to-do adolescent school girls towards breast feeding. Indian Pediatr. 1990;27(12):1281-5.

34. Swanson V, Power K, Kaur B, Carter H, Shepherd K. The impact of knowledge and social influences on adolescents' breast-feeding beliefs and intentions. Public Health Nutr. 2006;9(03):297-305.

35. Hadley C, Lindstrom D, Belachew T, Tessema F. Ethiopian adolescents' attitudes and expectations deviate from current infant and young child feeding recommendations. J Adolesc Health. 2008;43(3):253-9.

36. Giles M, Connor S, McClenahan C, Mallett J, Stewart-Knox B, Wright M. Measuring young people's attitudes to breastfeeding using the Theory of Planned Behaviour. J Public Health (Oxf). 2007:29(1):17-26.

37. Ojofeitimi EO, Owolabi OO, Eni-Olorunda J, Adesina OF, Esimai OA. Promotion of exclusive breastfeeding (EBF): the need to focus on the adolescents. Nutr Health. 2001;15(1):55-62.

38. Forrester IT, Wheelock G, Warren AP. Assessment of students' attitudes toward breastfeeding. J Hum Lact. 1997;13(1):33-7.

39. Seidel AK, Schetzina KE, Freeman SC, Coulter MM, Colgrove NJ. Comparison of breast-feeding knowledge, attitudes, and beliefs before and after educational intervention for rural Appalachian high school students. South Med J. 2013;106(3):224-9

40. Greene J, Stewart-Knox B, Wright M. Feeding preferences and attitudes to breastfeeding and its promotion among teenagers in Northern Ireland. J Hum Lact. 2003;19(1):57-65.

41. Yeo S, Mulholland PM, Hirayama M, Breck S. Cultural views of breastfeeding among high-school female students in Japan and the United States: a survey. J Hum Lact. 1994;10(1):25-30.

42. Zeller CL. Effects of education on breastfeeding knowledge and attitudes among middle school students. Health Educ J. 2016;75(4):501-10.

43. Spear HJ. College students' experiences and attitudes regarding middle and high school-based breastfeeding education. J Sch Nurs. 2007;23(5):276-82.

44. Tjiang L, Binns C. Indonesian students' knowledge of breastfeeding. Breastfeed Rev. 2001:9(2):5-9.

45. Bomba AK, Chang Y, Knight KB, Tidwell DK, Wachter K, Endo S, West CK. College students' attitudes regarding infant feeding practices. J Fam Consum Sci. 2009;101(1):25-9.

46. Costa M, Diniz-Santos DR, Santana JS, Silva LR. The impact of an educational intervention on breastfeeding. Health Educ. 2006;106(4):309-14.

47. Bottaro SM, Giugliani ER. Effectiveness of an intervention to improve breastfeeding knowledge and attitudes among fifth-grade children in Brazil. J Hum Lact. 2009;25(3):325-32.

48. Frew JR, Taylor JS. First steps: a program for medical students to teach high school students about breastfeeding. Med Health R I. 2005;88(2):48-50.

49. Giles M, McClenahan C, Armour C, Millar S, Rae G, Mallett J, Stewart-Knox B. Evaluation of a theory of planned behaviour-based breastfeeding intervention in Northern Irish Schools using a randomized cluster design. $\mathrm{Br} J$ Health Psychol. 2014;19(1):16-35.

50. Kim Y. The effects of a breastfeeding campaign on adolescent Korean women. Pediatr Nurs. 1998;24(3):235-40.

51. Lockey R, Hart A. Addressing inequalities in health: the breasts benefits project. Br J Midwifery. 2003;11(5):281-7.

52. Martens PJ. The effect of breastfeeding education on adolescent beliefs and attitudes: a randomized school intervention in the Canadian Ojibwa community of Sagkeeng. J Hum Lact. 2001;17(3):245-55.
53. Walsh A, Moseley J, Jackson W. The effects of an infant-feeding classroom activity on the breast-feeding knowledge and intentions of adolescents. J Sch Nurs. 2008;24(3):164-9.

54. Giles M, Millar S, Armour C, McClenahan C, Mallett J, Stewart-Knox B. Promoting positive attitudes to breastfeeding: the development and evaluation of a theory-based intervention with school children involving a cluster randomised controlled trial. Matern Child Nutr. 2015;11(4):656-72.

55. Bailey J, Shepherd R. An intervention to improve adolescents' views on breastfeeding. Health Psychology Update. 2007;16(4):53-61.

56. Ho YJ, McGrath JM. Effectiveness of a breastfeeding intervention on knowledge and attitudes among high school students in Taiwan. J Obstet Gynecol Neonatal Nurs. 2016;45(1):71-7.

57. November L. Baby milk challenge: changing secondary school students' attitudes to breastfeeding. Br J Midwifery. 2013;21(11):775-81.

\section{Submit your next manuscript to BioMed Central and we will help you at every step:}

- We accept pre-submission inquiries

- Our selector tool helps you to find the most relevant journal

- We provide round the clock customer support

- Convenient online submission

- Thorough peer review

- Inclusion in PubMed and all major indexing services

- Maximum visibility for your research

Submit your manuscript at www.biomedcentral.com/submit 